\title{
C-Arm Flat Detector CT Parenchymal Blood Volume Thresholds for Identification of Infarcted Parenchyma in the Neurointerventional Suite
}

\author{
M. Kamran and J.V. Byrne
}

\begin{abstract}
BACKGROUND AND PURPOSE: C-arm flat detector CT parenchymal blood volume imaging allows functional assessment of the brain parenchyma in the neurointerventional suite. This study aimed to determine the optimal C-arm flat detector CT parenchymal blood volume thresholds for demarcating irreversibly infarcted brain parenchyma by using areas of restricted diffusion on MR imaging as a surrogate marker for infarction.
\end{abstract}

MATERIALS AND METHODS: Twenty-six patients with delayed cerebral ischemia following aneurysmal SAH underwent research C-arm CT parenchymal blood volume scans by using a biplane angiography system and contemporaneous MR imaging. Infarct and peri-infarct tissue VOIs and their homologous VOIs in the contralateral uninvolved hemisphere were delineated on the basis of the review of DWI, PWI, and ADC images. Voxel-based receiver operating characteristic curve analysis was performed to estimate the optimal absolute and normalized parenchymal blood volume values for demarcating the infarct voxels.

RESULTS: For 12 patients with areas of restricted diffusion (infarct volume, $6.38 \pm 7.09 \mathrm{~mL}$; peri-infarct tissue volume, $22.89 \pm 21.76 \mathrm{~mL}$ ) based on the voxel-based receiver operating characteristic curve analysis, optimal absolute and normalized parenchymal blood volume thresholds for infarction were $2.49 \mathrm{~mL} / 100 \mathrm{~g}$ (area under curve, 0.76; sensitivity, 0.69; specificity, 0.71 ) and 0.67 (area under curve, 0.77 ; sensitivity, 0.69; specificity, 0.72), respectively ( $P$ value $<.01)$. For the moderate-to-severely ischemic peri-infarct zone, mean parenchymal blood volume values of the involved hemisphere VOls were lower compared with the uninvolved hemisphere vOls $(P$ value $<.01)$. However, for the mild-to-moderately ischemic peri-infarct zone, there was no statistically significant difference between the mean parenchymal blood volume values of the involved and uninvolved hemisphere VOls $(P$ value $>.05)$.

CONCLUSIONS: C-arm flat detector CT parenchymal blood volume maps in conjunction with optimal thresholds are sensitive and specific for the estimation of irreversibly infarcted parenchyma. Parenchymal blood volume maps allow reliable detection of moderateto-severe ischemia; however, the potential for underestimation of mild-to-moderate ischemia exists.

ABBREVIATIONS: $\mathrm{DCI}=$ delayed cerebral ischemia; $\mathrm{FDCT}=$ flat detector $\mathrm{CT} ; \mathrm{PBV}=$ parenchymal blood volume; $\mathrm{ROC}=$ receiver operating characteristic

$\mathbf{F}$

lat detector equipped C-arm angiographic systems used in the neurointerventional suite are now capable of measuring parenchymal blood volume (PBV) by using a specially designed scan protocol. ${ }^{1}$ Initial reports document agreement between C-arm flat detector CT (FDCT) PBV and CT perfusion-derived cerebral blood volume in both canines and humans. ${ }^{2,3}$ The availability of PBV maps in the interventional suite is an important develop-

Received November 18, 2014; accepted after revision February 11, 2015.

From the Nuffield Department of Surgical Sciences, University of Oxford, John Radcliffe Hospital, Headington, Oxford, United Kingdom.

M.K. is funded by the Rhodes Trust, and J.V.B. is supported by the Oxford Biomedical Research Centre.

Please address correspondence to Mudassar Kamran, MD, MSc, Nuffield Department of Surgical Sciences, University of Oxford, Room 6607, Level 6, John Radcliffe Hospital, Headington, Oxford, UK, OX3 9DU; e-mail: m.kamran@oxon.org

http://dx.doi.org/10.3174/ajnr.A4339 ment and has potential implications in the management of patients with ischemic cerebrovascular disease and their selection for revascularization procedures. ${ }^{4}$

Areas of reduced PBV on the initial C-arm FDCT PBV maps have been shown to correlate with the infarct volume on follow-up imaging in patients with acute middle cerebral artery occlusion. ${ }^{4} \mathrm{C}$-arm FDCT PBV and the CTP-derived CBV are not identical. However, an equivalence between the 2 is usually inferred if a steady-state contrast concentration is achieved in the cerebral vasculature during the $\mathrm{C}$-arm FDCT PBV scan; in the absence of an ideal steady-state, the PBV may have combined CBF and CBV weighting or even preferential CBF weighting. ${ }^{1,5}$ For CTP, although debate exists as to which perfusion parameter best defines irreversible infarction, thresholds for various parameters corresponding to acute DWI abnormality and infarct core have been reported. ${ }^{6-8}$ No such thresholds have so far been investigated for C-arm CT PBV. 
The aims of this study were: 1) to determine the optimal Carm CT PBV thresholds for irreversibly infarcted parenchyma by using contemporaneous MR DWI in patients with delayed cerebral ischemia (DCI) following aneurysmal subarachnoid hemorrhage, and 2) to investigate whether the C-arm CT PBV values in the peri-infarct ischemic zone allow reliable demarcation of the ischemic tissue.

\section{MATERIALS AND METHODS}

With local research ethics committee approval, a series of patients ( $n=26$ ) with aneurysmal SAH meeting the inclusion and exclusion criteria was enrolled in this prospective study. Inclusion criteria were patients older than 18 years of age with documented aneurysmal SAH who subsequently developed symptoms and signs suggesting DCI and requiring confirmatory imaging. The diagnosis of DCI was based on clinical assessment by the multidisciplinary neuro-intensive care unit team and was defined as clinical deterioration (focal neurologic deficits or reduced level of consciousness) lasting 2 hours or longer with no evidence of rebleed or hydrocephalus on NCCT of the head and no other medical causes such as infections or metabolic disturbances. Exclusion criteria were the following: a history of allergy to iodinated contrast medium, renal impairment, pregnancy, or contraindications to MR imaging. All recruited patients had undergone aneurysm repair by endovascular coil embolization. Written informed consents were obtained from patients or, if they were unable to complete the consent process, from a family member or a designated surrogate as defined by the research ethics committee guidelines. All recruited patients underwent an MR imaging examination concurrent with the research C-arm CT scan by using the intravenous PBV protocol. The imaging protocols and postprocessing steps for the 2 imaging studies are described in the following sections.

\section{C-Arm FDCT Imaging}

C-arm FDCT scans were obtained on a flat panel-equipped biplane angiography system (Axiom Artis dBA; Siemens, Erlangen, Germany) in the neurointerventional suite. The imaging protocol included 2 rotational acquisitions: mask and contrast-enhanced runs. Projection data for each run were acquired by using the following parameters: 419 projection images, $0.5^{\circ}$ frame angle, $210^{\circ}$ angular scan range, 8 -second acquisition time, $0.36-\mu \mathrm{Gy}$ radiation dose per frame. The contrast-enhanced dataset was acquired following manually triggered intravenous injection of radiographic contrast material, iopamidol (Niopam 370; Bracco, Milan, Italy) by using a power injector (Medrad, Indianola, Pennsylvania). The injection parameters were the following: contrast concentration, 370-mg iodine $/ \mathrm{mL}$; volume, $80 \mathrm{~mL}$; injection rate, $4 \mathrm{~mL} / \mathrm{s}$ administered through the antecubital vein; injection pressure, 300 psi; and injection duration, 20 seconds. Passage of contrast material through the cerebral vasculature was monitored on 2D fluoroscopic images ( 2 frames/s), and the contrast-enhanced run was triggered when maximal opacification of the superior sagittal sinus was observed, as described previously by Struffert et al. ${ }^{3}$

Postprocessing of the data to generate color-coded PBV maps was performed on a dedicated research workstation (Leonardo;
Siemens) by using prototype software. The steps involved were the following: 1) coregistration of the 2 volumes, mask and contrast-enhanced; 2) subtraction of the mask volume from the contrast-enhanced volume; 3 ) normalization of the subtracted data with an input function automatically estimated from the histogram analysis of vascular tree; and 4) color-coding with a standard color look-up table to generate the color-coded PBV maps for visualization and interpretation. The final PBV values were expressed in units of milliliters $/ 1000 \mathrm{~mL}$ of cerebral tissue. PBV values for volume-of-interest analysis were converted to more commonly used units of milliliters/100 g by using a simple conversion factor that takes into account the cerebral tissue density $(1.05 \mathrm{~g} / \mathrm{mL})$.

\section{MR Imaging}

A standard MR image protocol comprising sagittal T1-weighted, axial T1-weighted, axial T2-weighted, diffusion-weighted, perfusion-weighted, and time-of-flight MR angiography sequences was performed on an Achieva 1.5T MR imaging unit (Philips Healthcare, Best, Netherlands). Details for the diffusion- and perfusionweighted sequences and the parameters used for these sequences are briefly discussed below.

DWI. DWI parameters were the following: TR, $2897 \mathrm{~ms}$; TE, 73 ms; FOV, $174 \times 249 \mathrm{~mm}$; reconstruction matrix, $144 \times 144$; sections, 25 ; section thickness, $5 \mathrm{~mm}$; voxel size, $1.74 \times 1.73 \times 5$ mm; b-values, 0, 1000; EPI factor, 51; scan duration, 34 seconds.

PWI. Perfusion data were acquired by using the dynamic susceptibility contrast technique with the following parameters: $\mathrm{T} 2{ }^{\star}$ weighted gradient-echo echo-planar sequence: TR, $2000 \mathrm{~ms}$; TE, $44 \mathrm{~ms}$; FOV, $248 \times 248 \mathrm{~mm}$; reconstruction matrix, $256 \times 256$; voxel size, $0.98 \times 0.98 \times 4 \mathrm{~mm}$; sensitivity encoding factor, 2.1 ; bandwidth, $806 \mathrm{~Hz} /$ pixel; scan duration, 1 minute 46 seconds. Paramagnetic contrast material was administered intravenously with an MR imaging-compatible power injector (Medrad, Indianola, Pennsylvania) at $5 \mathrm{~mL} / \mathrm{s}$ followed by a 10 -mL saline flush. Fifteen sections covering the whole brain were acquired at 50 time points following the injection of $0.2 \mathrm{mmol} / \mathrm{kg}$ of gadoteridol (ProHance; Bracco).

Postprocessing of the perfusion data was performed by using commercially available software (nordicICE perfusion package; NordicNeuroLab, Bergen, Norway). After motion correction of the raw data, we created perfusion maps by using the singular value deconvolution technique. The arterial input function to deconvolve the dynamic tissue response curve was automatically determined, and singular value decomposition regularization was achieved with the truncated singular value deconvolution approach (cutoff fraction, 0.20) and iterative thresholding (target oscillation index, 0.10). Semiquantitative output perfusion maps for clinical interpretation included cerebral blood flow, cerebral blood volume, mean transit time, and time to peak.

\section{Postprocessing and Quantitative Image Analysis}

C-arm CT PBV, PWI, apparent diffusion coefficient, and DWI were further processed by using the fMRI of the Brain Software Library tools. ${ }^{9}$ The Brain Extraction Tool (http://fsl.fmrib.ox. ac.uk/fsl/fslwiki/BET) was used to delete the nonbrain tissues 


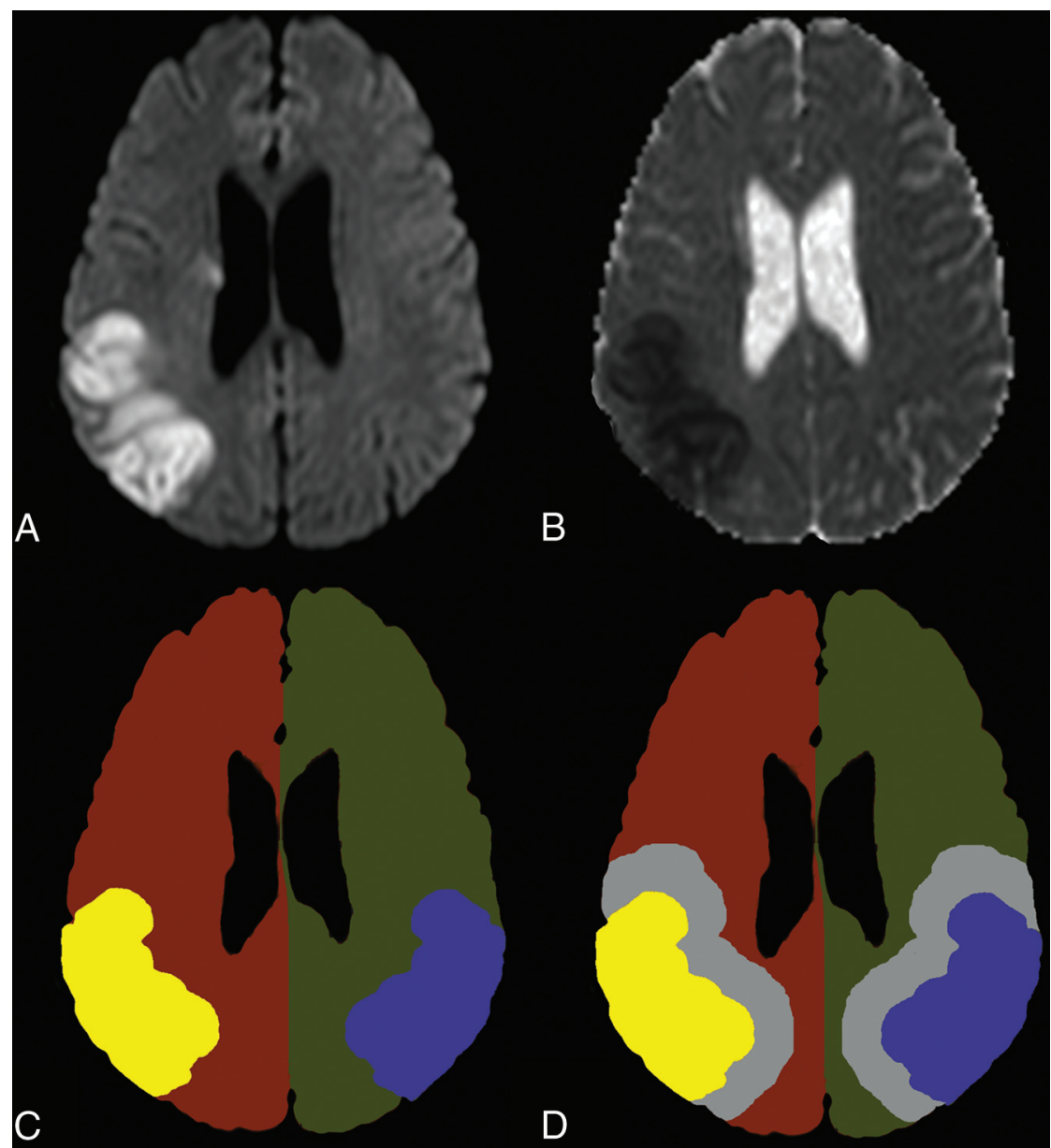

FIG 1. Binary masks used for VOI analysis. An area of restricted diffusion was identified on the $D W I$ and $A D C$ maps ( $A$ and $B$ ). Corresponding to the area of restricted diffusion (surrogate for infarcted tissue), a VOI mask (yellow) was created by a semiautomatic approach, along with a mirrored VOI mask (blue) in the contralateral normal hemisphere. Surrounding the VOI mask corresponding to the area of restricted diffusion, a peri-infarct tissue VOI mask (gray) was created on the basis of the review of PWI relative CBF maps. A mirrored VOI mask for the peri-infarct tissue in the contralateral normal hemisphere was constructed by transposing the peri-infarct tissue VOI mask. Hemispheric masks for the ipsilateral (orange) and contralateral hemisphere (green) were constructed for calculation of normalized PBV values.

from the images of interest followed by linear registration of the C-arm PBV maps and the MR-PWI, PWI, and ADC images for each patient to the T1-weighted average structural template brain volume in Montreal Neurological Institute 152 space with the fMRI of the Brain (FMRIB) Linear Image Registration Tool (FLIRT; http://www.fmrib.ox.ac.uk). ${ }^{10,11}$ Alignment of all coregistrations was visually confirmed in 3 orthogonal planes. Areas of infarction were identified on the basis of the review of DWI and ADC maps. 3D VOIs corresponding to the infarct core were defined on the DWI with a semiautomated approach by using MRIcro software (http://www.mccauslandcenter.sc.edu/mricro/ mricro/mricro.html). ${ }^{12}$ In the next step, the infarct core VOIs were grown to include the peri-infarct ischemic parenchyma identified as the region of asymmetrically reduced CBF $(<95 \%$ relative to the mean contralateral hemisphere $\mathrm{CBF}$ ) surrounding the infarct core defined on the DWI. From the grown VOIs that included both the infarct core and the peri-infarct ischemic tissue, the infarct core VOIs were subtracted, thus creating the subtraction VOIs defining only the peri-infarct ischemic tissue. In the contralateral normal hemisphere, mirrored homologous tissue VOIs were then created corresponding to the affected hemisphere infarct core and peri-infarct ischemic tissue VOIs. In a small subset of patients for whom bilateral areas of ischemia or infarction existed, substitute VOIs with comparable volume in the uninvolved adjacent parenchyma were created and used for comparison and normalization. All VOIs were subsequently transposed onto coregistered C-arm CT PBV maps. Figures 1 and 2 illustrate the VOI masks used for analysis. Mean, median, and SD of the absolute PBV values in all VOIs were measured by using FMRIB software maths (http://fsl.fmrib.ox.ac.uk/ fsl/fslwiki/Fslutils) and FMRIB software stats utilities (http://fsl.fmrib.ox.ac.uk/ fsl/fslwiki/Fslutils). Normalized PBV values were calculated by dividing the absolute PBV value for each VOI by the mean contralateral hemisphere PBV value obtained by using separate hemispheric masks.

\section{Statistical Analysis}

A paired $t$ test was performed to investigate whether mean PBV in the infarct tissue VOIs and the mean PBV in the contralateral uninvolved homologous tissue VOIs were statistically different and whether the mean PBV in peri-infarct ischemic parenchyma VOIs and the mean PBV in contralateral uninvolved homologous tissue VOIs were statistically different.

To test the predictive performance of $\mathrm{C}$-arm CT PBV maps across various thresholds for demarcating infarcted tissue, we performed voxel-based receiver operating characteristic (ROC) curve analysis. Sensitivity and specificity of PBV maps for demarcating the infarct voxels (DWI/ADC abnormality) from the noninfarct voxels across patients as a function of increasing PBV thresholds were computed. The accuracy of PBV maps in demarcating the infarct voxels (DWI/ADC abnormality) from the noninfarct voxels was determined from the area under curve statistic that ranges from 0.5 (no predictive value) to 1 (absolute predictive value). The ROC curve analysis was performed separately for the absolute and the normalized PBV values. The final absolute and normalized PBV thresholds for demarcating infarcted tissue were calculated as the optimal ROC curve points with equally attributed weights to sensitivity and specificity. Statistical analysis was performed by using GraphPad Prism software (GraphPad Software, San Diego, California).

\section{RESULTS}

\section{Study Population Characteristics}

Twenty-six patients (mean age, 55 years; 9 men, 17 women) entered the study. Patients' World Federation of Neurosurgical So- 


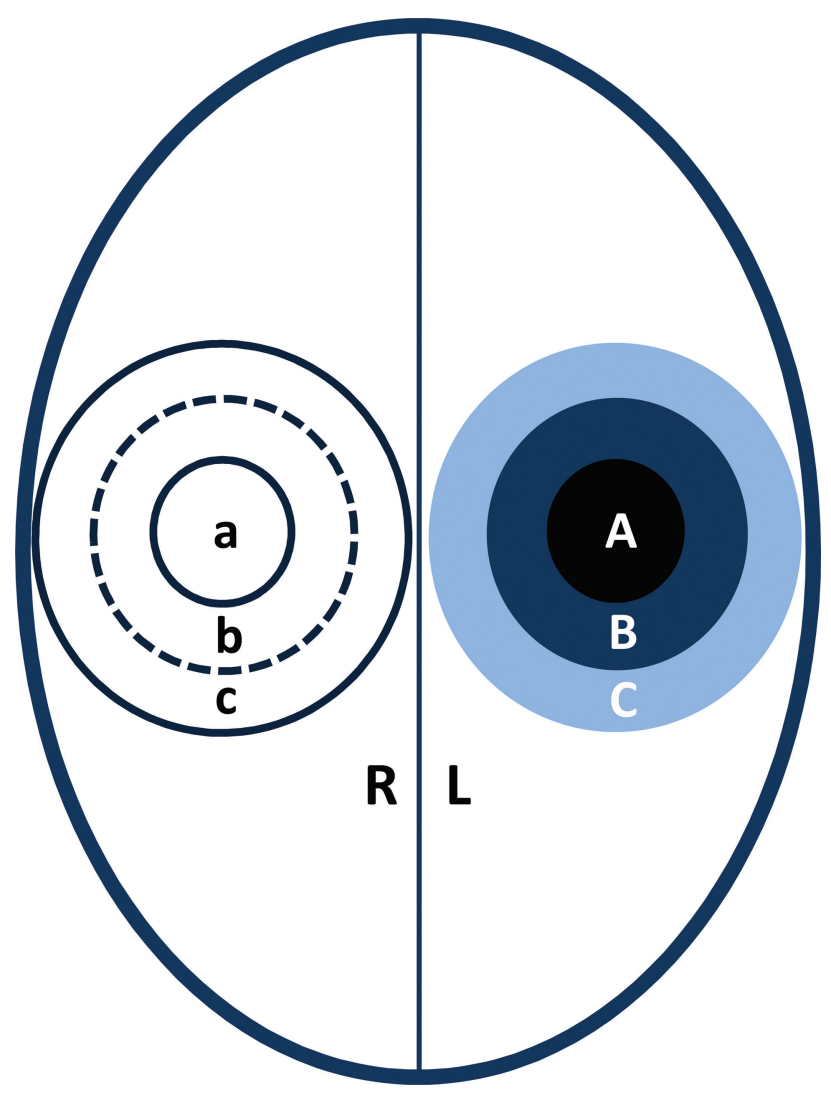

FIG 2. Schematic illustration of the infarct and peri-infarct VOIs. In the involved hemisphere (L), the infarct VOI (A) corresponds to the area of restricted diffusion (surrogate for infarction) on DWI and ADC images. Surrounding the infarct VOI, the peri-infarct zone $(B+C)$ corresponds to the region of asymmetrically reduced $\mathrm{MR}$ imaging $\mathrm{CBF}$ (CBF of $<95 \%$ relative to the mean contralateral hemisphere $C B F$ ). The peri-infarct zone $(B+C)$ was further subdivided into a mild-tomoderately ischemic zone ( $\mathrm{B}$; $\mathrm{CBF}$ of $75 \%-95 \%$, relative to the mean contralateral hemisphere $\mathrm{CBF}$ ) and a moderate-to-severely ischemic zone (C; $C B F$ of $<75 \%-95 \%$, relative to the mean contralateral hemisphere $\mathrm{CBF}$ ). Contralateral homologous tissue VOIs in the uninvolved hemisphere $(R)$ corresponding to infarct $(a)$ and peri-infarct $(b+c)$ zones were created by transposing the involved hemisphere vOls across the midline.

cieties grades at the time of admission to the hospital were the following: grade I in 16 (61\%), grade II in 7 (27\%), grade III in 2 (8\%), and grade IV in 1 patient (4\%). The onset of DCI after SAH varied from day 2 post-SAH to day 12 post-SAH (median, day 7 post-SAH). All patients received standard medical management according to the institutional protocol. Of the 26 patients, 8 (31\%) underwent chemical angioplasty with selective intra-arterial nimodipine administration, 2 (8\%) of whom received chemical angioplasty treatment twice. The mean duration between the $\mathrm{C}$-arm FDCT PBV scan and the MR imaging was 124 minutes. Of the 26 patients, 12 had areas of restricted diffusion on MR imaging. On these scans, 22 VOIs each for infarcted parenchyma, periinfarct tissue, and their contralateral homologous tissue were defined (total, $22 \times 4$ VOIs); The Table describes the patient characteristics and details of DWI lesions.

\section{Quantitative Image Analysis}

For the patients with areas of restricted diffusion on DWI, mean DWI lesion volume (infarcted parenchyma) was $6.38 \pm 7.09 \mathrm{~mL}$ (range, $0.91-26.5 \mathrm{~mL}$ ) and the mean peri-infarct ischemic tissue volume was $22.89 \pm 21.76 \mathrm{~mL}$ (range, $4.18-85.61 \mathrm{~mL}$ ).

Mean PBV values in the infarct VOIs were significantly lower compared with the mean PBV values in the mirrored contralateral uninvolved homologous tissue VOIs (paired $t$ test $P$ value $<.01$ ), as illustrated in the box-and-whisker plot (Fig 3A). Voxel-based ROC curve analysis was performed for both absolute and normalized C-arm CT PBV volumetric parametric maps, and the resulting ROC curves are shown in Fig 4. The optimal absolute C-arm CT PBV threshold identifying the infarcted parenchyma by using DWI abnormality as a marker was $2.49 \mathrm{~mL} / 100 \mathrm{~g}$. The corresponding area under the curve, sensitivity, and specificity were 76.4\% (95\% CI, 76.1-76.7), 69.4\% (95\% CI, 68.9-69.8), and 70.7\% (95\% CI, 70.2-71.1) ( $P$ value $<.01)$, respectively. The optimal normalized C-arm CT PBV threshold was 0.67 . The corresponding area under the curve, sensitivity, and specificity were 76.9\% (95\% CI, 76.6-77.1), 69.4\% (95\% CI, 69.0-69.7), and $72.2 \%$ (95\% CI, 71.8-72.5) ( $P$ value $<.01)$, respectively.

A paired-samples $t$ test conducted to compare the mean PBV values for peri-infarct ischemic tissue VOIs and the mean PBV values for corresponding mirrored VOIs in the contralateral uninvolved hemisphere showed no significant difference between the mean PBV values for the 2 groups of VOIs $(P>.05)$. Given this finding, the analysis was extended by dividing each of the peri-infarct VOIs into 2 segments based on the review of MR imaging relative CBF maps: 1) moderate-to-severely ischemic peri-infarct zone with $\mathrm{MR} \mathrm{CBF}$ of $<75 \%$ relative to the mean contralateral hemisphere $\mathrm{CBF}$, and 2) a mild-to-moderately ischemic peri-infarct zone with $\mathrm{MR} C \mathrm{CBF}$ of $75 \%-95 \%$ relative to the mean contralateral hemisphere CBF (Fig 2). VOIs and binary masks for the 2 zones were created for both the involved and the uninvolved hemisphere by following the same approach as used earlier in the analysis, followed by the application of the binary masks to the C-arm CT PBV maps. Mean PBV values for the 2 sets of VOIs corresponding to the mild-to-moderate and the moderate-to-severely ischemic peri-infarct zones were then compared with their contralateral homologous tissue VOIs by using the paired-samples $t$ test. For the moderate-to-severely ischemic periinfarct zone, the mean PBV values of the involved hemisphere VOIs were statistically significantly lower compared with the uninvolved hemisphere VOIs (Fig $3 B, P$ value $<.01$ ). However, for the mild-to-moderately ischemic peri-infarct zone, there was no statistically significant difference between the mean PBV values of the involved and uninvolved hemisphere VOIs (Fig $3 C, P$ value $>$ $.05)$.

\section{DISCUSSION}

This study reports optimal absolute and normalized C-arm CT PBV threshold values for demarcating irreversibly infarcted brain parenchyma in the neurointerventional suite.

In determining the PBV threshold for infarcted brain parenchyma, areas of restricted diffusion identified on the review of DWI and ADC maps were used as a marker for irreversibly infarcted tissue. Although DWI abnormality is not perfectly specific for infarct core, it is highly accurate and is an accepted standard for both clinical care and research analysis. ${ }^{13-15}$ In our analysis, we used 3D VOIs, defined semiautomatically for the DWI abnormal- 


\begin{tabular}{|c|c|c|c|c|c|c|c|c|}
\hline $\begin{array}{c}\text { Patient } \\
\text { No. }\end{array}$ & $\begin{array}{l}\text { Age } \\
\text { (yr) }\end{array}$ & Sex & $\begin{array}{l}\text { Aneurysm } \\
\text { Location }\end{array}$ & $\begin{array}{l}\text { Aneurysm } \\
\text { Size }(\mathrm{mm})\end{array}$ & $\begin{array}{l}\text { WFNS } \\
\text { Grade }\end{array}$ & $\begin{array}{l}\text { Fisher } \\
\text { Grade }\end{array}$ & $\begin{array}{c}\text { DWI } \\
\text { Deficits }\end{array}$ & DWI Deficits Distribution \\
\hline 1 & 47 & M & Anterior communicating artery & $6 \times 4$ & 1 & 3 & + & Right frontal operculum; right centrum semiovale \\
\hline 2 & 55 & $\mathrm{~F}$ & Left anterior cerebral artery & $12 \times 10$ & III & 4 & - & - \\
\hline 3 & 61 & $\mathrm{~F}$ & Right posterior communicating artery & $8 \times 5$ & ॥ & 3 & - & - \\
\hline 4 & 49 & $\mathrm{~F}$ & Anterior communicating artery & $10 \times 7$ & $\mathrm{I}$ & 3 & - & - \\
\hline 5 & 68 & $\mathrm{~F}$ & Right posterior communicating artery & $10 \times 6$ & II & 4 & - & - \\
\hline 6 & 71 & $\mathrm{~F}$ & Anterior communicating artery & $6 \times 5$ & 1 & 4 & + & Right frontal and left posterior frontal lobes \\
\hline 7 & 46 & M & Right middle cerebral artery & $4 \times 3$ & III & 3 & + & Bifrontal and posterior left parietal lobes \\
\hline 8 & 77 & $\mathrm{~F}$ & Left anterior cerebral artery & $6 \times 4$ & 1 & 4 & - & - \\
\hline 9 & 46 & M & Right posterior communicating artery & $4 \times 4$ & I & 4 & - & - \\
\hline 10 & 50 & $\mathrm{~F}$ & Right internal carotid artery & $3 \times 3$ & I & 3 & + & Right frontal lobe \\
\hline 11 & 53 & $\mathrm{~F}$ & Basilar artery tip & $7 \times 6$ & II & 3 & + & Left frontal lobe \\
\hline 12 & 55 & M & Right posterior communicating artery & $5 \times 3$ & II & 4 & - & - \\
\hline 13 & 55 & $\mathrm{~F}$ & Left posterior communicating artery & $4 \times 4$ & II & 4 & - & - \\
\hline 14 & 69 & $\mathrm{~F}$ & Left anterior cerebral artery & $11 \times 7$ & $\mathrm{I}$ & 4 & + & Bifrontal lobes \\
\hline 15 & 66 & M & Anterior communicating artery & $7 \times 5$ & I & $0^{\mathrm{a}}$ & - & - \\
\hline 16 & 45 & M & Anterior communicating artery & $15 \times 8$ & II & 4 & + & Right parietal lobe and corpus striatum \\
\hline 17 & 66 & M & Right middle cerebral artery & $8 \times 3$ & IV & 4 & + & Right parietal lobe and Sylvian fissure \\
\hline 18 & 30 & M & Anterior communicating artery & $7 \times 4$ & I & 3 & + & Left frontal lobe \\
\hline 19 & 39 & $\mathrm{~F}$ & Right posterior communicating artery & $5 \times 3.5$ & $\mathrm{I}$ & 4 & + & Right parietal lobe \\
\hline 20 & 50 & $\mathrm{~F}$ & Anterior communicating artery & $4 \times 3.5$ & $\mathrm{I}$ & 4 & - & - \\
\hline 21 & 69 & M & Right posterior communicating artery & $7 \times 4$ & I & 4 & - & - \\
\hline 22 & 39 & $\mathrm{~F}$ & Right posterior communicating artery & $10 \times 9$ & 1 & 4 & + & Right frontal lobe \\
\hline 23 & 52 & $\mathrm{~F}$ & Right posterior communicating artery & $5 \times 5$ & 1 & 4 & + & - \\
\hline 24 & 54 & $\mathrm{~F}$ & Left posterior communicating artery & $8 \times 5$ & 1 & 3 & + & - \\
\hline 25 & 58 & $\mathrm{~F}$ & Right anterior cerebral artery & $5 \times 4$ & 1 & 3 & + & Right frontal lobe \\
\hline 26 & 60 & $\mathrm{~F}$ & Left pericallosal artery & $12 \times 8$ & II & 4 & - & - \\
\hline
\end{tabular}

Note:-WFNS indicates World Federation of Neurological Societies; +, present; - , absent.

${ }^{a} \mathrm{NCCT}$ of the head was negative for $\mathrm{SAH}$, which was later confirmed on lumbar puncture.
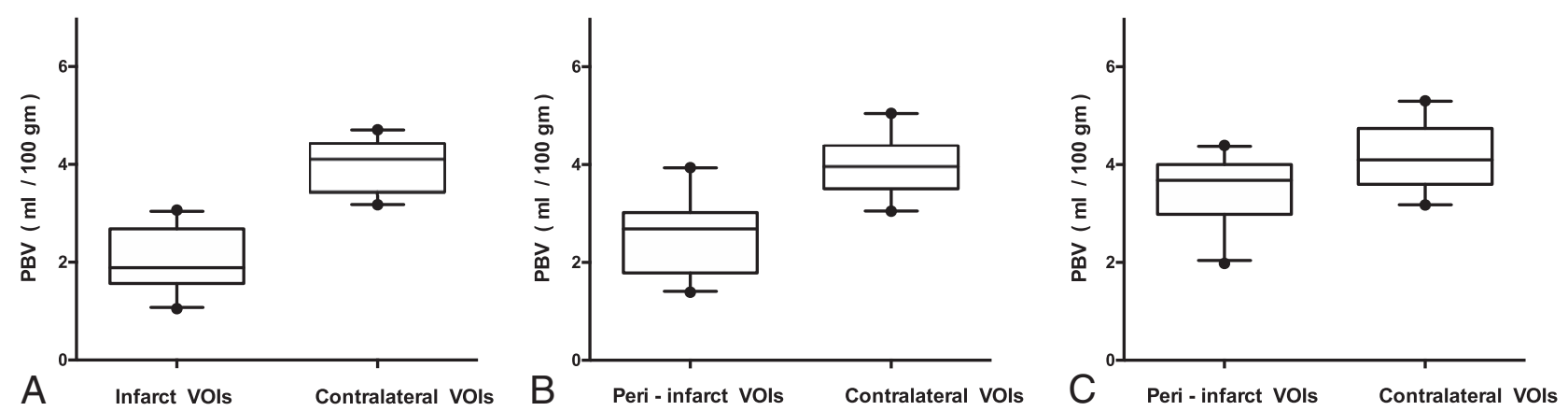

FIG 3. Box-and-whisker plots showing PBV (mean and SD) in the infarct VOls - that is, areas of restricted diffusion (A), moderate-to-severely ischemic peri-infarct VOls (relative CBF of $<75 \%$ ) (B), mild-to-moderately ischemic, peri-infarct VOls (relative CBF of $75 \%-95 \%$ ) (C), and the PBV in the corresponding mirrored contralateral homologous tissue VOls.

ity (infarcted parenchyma), which, along with mirrored contralateral homologous tissue VOIs, were transposed onto the coregistered C-arm CT PBV maps before performing the ROC curve analysis. This objective approach toward image analysis reduces any bias that may be introduced due to subjective differences in image display, such as color look-up tables for PBV maps, window settings, and pixel conspicuity, which can be sources of error for manual segmentation. ${ }^{7}$

That any perfusion study represents a "snapshot in time" and the cerebral hemodynamics and thresholds for ischemic damage may vary with time are issues well-recognized from CTP imaging of acute brain ischemia. For acute stroke imaging, this limitation may be addressed through stratifying data by duration postictus. ${ }^{7}$ However, unlike acute ischemic stroke, the complex pathophysiology of cerebral vasospasm and resulting hemodynamic alterations make it difficult to define a specific time-ofonset for DCI secondary to vasospasm. Moreover, the limited number of patients in our study precluded any such analysis, which could theoretically be performed by using an arbitrary time point such as the development of neurologic deficits in patients post-SAH.

An important factor that may introduce variability in C-arm CT PBV measurements and consequently shift the optimal PBV thresholds for infarction is how well the steady-state assumption is met. For calculation of C-arm CT PBV, the intravenously administered iodinated contrast material is assumed to have reached a steady-state concentration in the cerebral vasculature (contrast concentration in arteries $=$ contrast concentration in tissues $=$ contrast concentration in veins), maintained for the duration of the scan. ${ }^{1}$ If this assumption is fully met, the C-arm CT PBV would be equivalent to the CBV derived from dynamic perfusion imaging modalities. However, an ideal steady-state may not be achieved in practice for the clinical scans, and the contrast profile in the cerebral vasculature may show intersubject differences due to various factors such as recirculation of contrast, hemodynamic perturbation effect, and slow bolus 


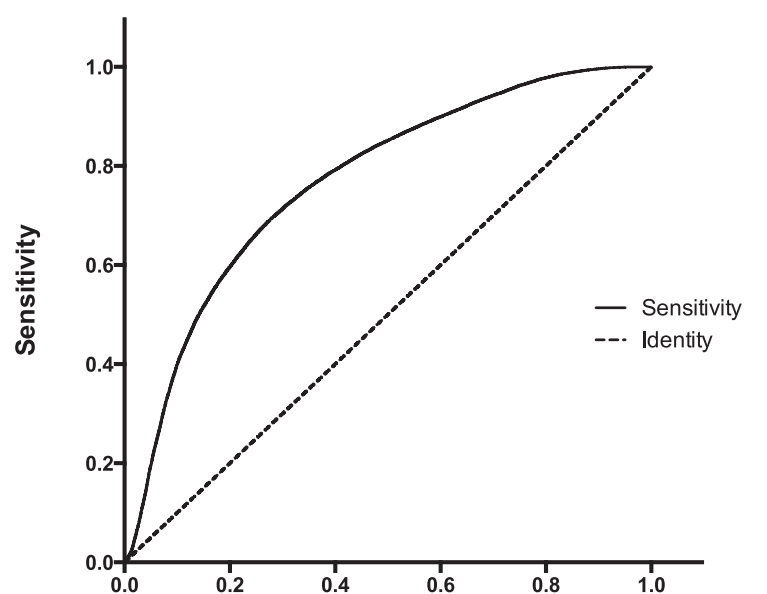

A

1 - Specificity

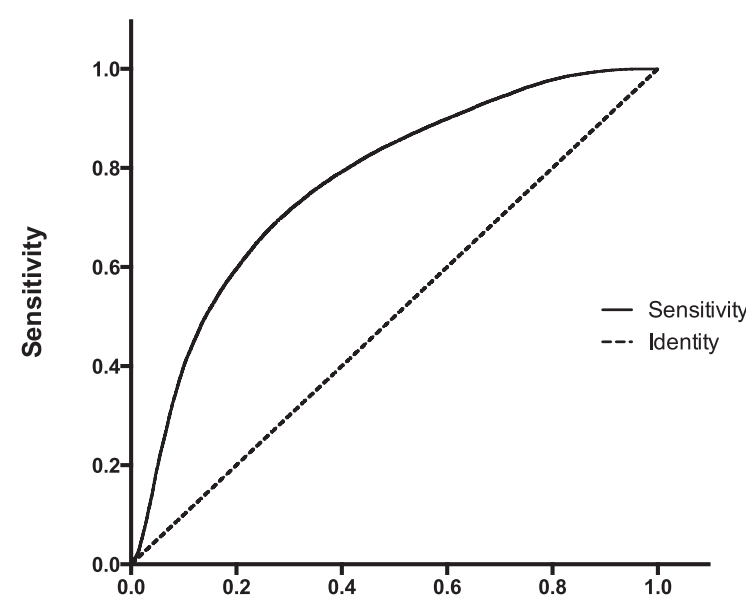

B

FIG 4. ROC curves for absolute and normalized PBV. Voxel-based ROC curves for absolute PBV $(A)$ and normalized PBV $(B)$, with near-identical configurations. Absolute and normalized PBV thresholds for demarcating the areas of restricted diffusion were chosen by defining the optimal point on the ROC curve with equally attributed weights to sensitivity and specificity.

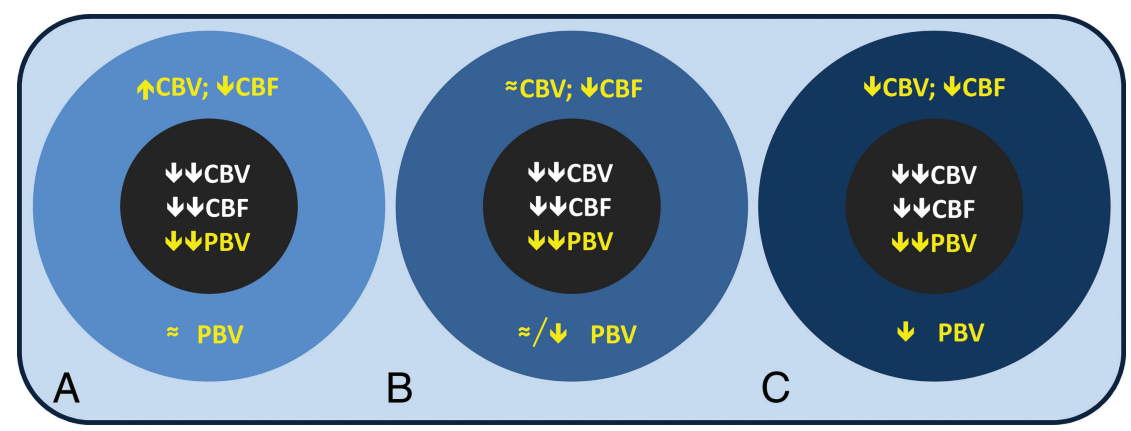

FIG 5. A theoretic understanding of $C$-arm $C T$ PBV at various degrees of ischemia. In the infarcted tissue (black central zone in $A-C$ ), a matched decrease in $C B V$ and $C B F$ with consequent reduced PBV is observed, given its combined blood flow and blood volume weightings. In mild ischemia $(A), C B F$ is reduced while there is a compensatory autoregulatory increase in CBV; consequently, $\mathrm{PBV}$ values may be maintained. In moderate ischemia $(B)$, the $C B F$ reduces further, and despite autoregulation, CBV may not increase; consequently PBV values may be in the lower part of normal range or begin to show mild reduction. In severe ischemia bordering infarction (C), both CBV and CBF would be decreased with consequent reduction in PBV.

transit in the setting of low cardiac output and multifocal vasospasm. ${ }^{16}$

Preferential contrast wash-in or washout during the acquisition of fill run data for the C-arm CT PBV study (8 seconds) may result in variable blood flow weighting to the calculated PBV. In a separate analysis for the same cohort (subject of another publication), we found that for clinical examinations, the C-arm CT PBV has $\approx 60 \% \mathrm{CBF}$ and $40 \% \mathrm{CBV}$ weighting, with similar supporting observations for multidetector CT. ${ }^{5,17}$ Thus, differences in contrast dynamics can result in differences in blood flow and blood volume weighting to PBV, which can translate into differences in final PBV measurements. Assuming identical contrast dynamics or an ideal steady-state in all patients despite the differences in their hemodynamic physiology may overlook the blood flow weighting to C-arm CT PBV, particularly in cases in which the time-attenuation curves depart from the steady-state, thus potentially resulting in overestimation (or underestimation) of irreversibly infarcted parenchyma when using a specific threshold. This limitation can be overcome by a simple ROI-based timeattenuation-curve analysis of the raw $\mathrm{C}$-arm $\mathrm{CT}$ projection data for the clinical PBV scans, which can provide an estimate of how adequately the steady-state contrast concentration was achieved during a particular study.

Our results indicate that the measured PBV values in the peri-infarct ischemic tissue may or may not show reduction, depending on the severity of the ischemic insult. We found that in moderate-to-severely ischemic peri-infarct tissue (relative MR $\mathrm{CBF},<75 \%$ ), the PBV measurements showed statistically significant reduction compared with the contralateral uninvolved homologous tissue. However, PBV values in the mild-tomoderately ischemic peri-infarct parenchyma (relative MR CBF, 75\%-95\%) were not significantly reduced relative to the contralateral uninvolved homologous tissue. The peri-infarct ischemic zone we identified for analysis is conceptually similar to the penumbra region seen in the setting of acute ischemic stroke. These findings are interesting and can be explained by the combined blood volume and blood flow weighting to C-arm CT PBV. CT perfusion reveals that in the setting of acute ischemic stroke, the infarcted tissue shows a matched decrease in $\mathrm{CBV}$ and CBF. ${ }^{18,19}$ However, the ischemic tissue surrounding the infarct core has only reduced CBF, while the CBV may be maintained or elevated secondary to autoregulatory hemodynamics whereby precapillary vessels dilate in response to decreased cerebral perfusion pressure. ${ }^{19}$ In the setting of severe or prolonged ischemia, the autoregulatory mechanisms fail, leading to eventual reduction in $\mathrm{CBV}$. The degree to which the $\mathrm{CBF}$ is reduced and whether the $\mathrm{CBV}$ is elevated, maintained, or reduced are thus functions of the severity of the ischemic insult. In a separate analysis for the same cohort, we found that with combined blood volume and blood flow weighting (subject of another publication), C-arm CT PBV behaves as a composite parameter. Thus, in the irreversibly in- 
farcted tissue, due to an underlying matched decrease in CBV and $\mathrm{CBF}$, we see a definitive reduction in PBV (Fig $3 A$ ). In the periinfarct ischemic region of reduced $\mathrm{CBF}, \mathrm{PBV}$ would be maintained when the ischemic insult is less severe and the autoregulatory mechanisms are intact and effective in increasing the regional CBV (Fig 3C). On the contrary, PBV measurements would be reduced if the ischemia is prolonged or worsens in severity with consequent impaired autoregulation, ineffective in increasing or maintaining the CBV (Fig 3B). Therefore, C-arm CT PBV measurements would reliably detect moderate-to-severe ischemia but can potentially underestimate mild-to-moderate ischemia.

We recognize that $\mathrm{C}$-arm $\mathrm{CT}$ PBV imaging cannot replace DWI, which is highly accurate in the demarcation of irreversibly infarcted brain parenchyma. However, its availability in the neurointerventional suite is a unique advantage with implications for management of acute cerebrovascular ischemia. Struffert et $\mathrm{al}^{4}$ proposed that PBV maps may potentially be used to guide revascularization procedures through their ability to monitor the brain viability. In this regard, a single $\mathrm{C}$-arm $\mathrm{CT}$ study can provide both the anatomic and physiologic data for patients with acute stroke in the interventional suite without the need to transfer patients to CT or MR imaging facilities. For example, the C-arm CT softtissue images can reliably exclude intracranial hemorrhage, the angiographic reconstructions can help evaluate proximal vessel occlusion, and the PBV maps provide patient-specific physiologic data that can potentially be used, instead of the more generalized and nonspecific time criteria, in selecting appropriate patients for acute stroke interventions, particularly when a significant delay exists between initial imaging and patient arrival in the angiography suite. ${ }^{4,20}$ Similarly, in the patients with aneurysmal SAH who develop delayed clinical deterioration, the PBV maps can be used to determine the hemodynamic significance of vessel narrowing detected on catheter angiography and to identify any ongoing ischemia or established infarcts, thus guiding the angioplasty procedures. For these applications, extended craniocaudal coverage allowing whole-brain PBV measurements would be particularly beneficial - for example, the changes related to vasospasm may be multifocal or global in nature and the acute ischemic stroke may be in a distribution located outside the traditional supratentorial coverage of CTP and may thus be better evaluated with extended craniocaudal coverage of C-arm CT PBV.

For CTP and PWI, thresholds of various perfusion parameters have been studied to demarcate the irreversibly infarcted parenchyma, and it is widely recognized that the generalizability of any specific threshold may be limited secondary to marked differences across different algorithms and postprocessing software. ${ }^{17,21}$ For C-arm CT PBV measurement, currently only 1 commercially available application (syngo Neuro PBV IR; Siemens) exists. It is conceivable that in the future other similar postprocessing platforms will become available, in which case the validation of thresholds reported in this study across different platforms would be imperative.

The mean interval between the C-arm CT and MR imaging examinations in this study was 124 minutes, a duration during which the hemodynamics of the parenchymal areas included in the analysis may have altered; this change could result in an overor underestimation of the reported thresholds. The observations in this study should therefore be interpreted allowing for this limitation. Additionally, optimal PBV thresholds reported in this study were assessed for whole-brain tissue VOIs. However, differences between the gray and white matter exist regarding cellular composition, metabolism, the normal perfusion parameter values, and their neurochemical responses to ischemia. ${ }^{22-24}$ Tissuespecific infarction thresholds may thus be more informative in predicting the tissue outcomes in the setting of cerebrovascular ischemia. ${ }^{25}$ In our cohort, the limited number of patients and DWI lesions precluded such tissue-specific analysis to explore the differential variability of gray and white matter to ischemia. Future studies, including a larger number of patients with cerebral ischemia, may help in addressing this issue.

\section{CONCLUSIONS}

This study reports the following: 1) the optimal absolute and normalized C-arm CT PBV threshold values for demarcation of the irreversibly infarcted parenchyma in the neurointerventional suite; and 2) C-arm CT PBV maps reliably detect moderate-tosevere ischemia but can potentially underestimate the mild-tomoderate ischemia with intact autoregulatory mechanisms, likely related to its combined blood flow and blood volume weighting. Optimal C-arm CT PBV thresholds for infarction have not been studied previously and are of value in providing an objective criterion for the demarcation of irreversibly infarcted parenchyma. An accurate assessment of the brain viability thus obtained has implications for the management of acute brain ischemia.

\section{ACKNOWLEDGMENTS}

We thank Siemens for hardware and software support. We are grateful to the members and staff of the neuroradiology department, John Radcliffe Hospital, Oxford, where all the imaging data were acquired for this study.

Disclosures: Mudassar Kamran—RELATED: Grant: Siemens, ${ }^{\star}$ Comments: a research grant was made to the University of Oxford. In addition, Siemens provided the hardware and software support for the research scans. James V. Byrne-RELATED: Grant: Siemens, ${ }^{*}$ Comments: a small one-off grant to support work indirectly related to the project reported in this article; OTHER RELATIONSHIPS: historical scientific advisory board member for Siemens, for which travel expenses and so forth were paid. *Money paid to the institution.

\section{REFERENCES}

1. Zellerhoff M, Deuerling-Zheng Y, Strother CM, et al. Measurement of cerebral blood volume using angiographic $\mathrm{C}$-arm systems. Proc SPIE 2009;7262:72620H; doi:10.1117/12.812097

2. Ahmed AS, Zellerhoff M, Strother CM, et al. C-arm CT measurement of cerebral blood volume: an experimental study in canines. AJNR Am J Neuroradiol 2009;30:917-22

3. Struffert T, Deuerling-Zheng Y, Kloska S, et al. Cerebral blood volume imaging by flat detector computed tomography in comparison to conventional multislice perfusion CT. Eur Radiol 2011;21:882-89

4. Struffert T, Deuerling-Zheng Y, Engelhorn T, et al. Feasibility of cerebral blood volume mapping by flat panel detector $\mathrm{CT}$ in the angiography suite: first experience in patients with acute middle cerebral artery occlusions. AJNR Am J Neuroradiol 2012;33:618-25

5. Sharma M, Fox AJ, Symons $S$, et al. CT angiographic source images: flow- or volume-weighted? AJNR Am J Neuroradiol 2011;32:359-64

6. Christensen S, Mouridsen $\mathrm{K}, \mathrm{Wu} \mathrm{O}$, et al. Comparison of 10 perfusion MRI parameters in 97 sub-6-hour stroke patients using voxelbased receiver operating characteristics analysis. Stroke 2009;40: 2055-61 
7. Kamalian S, Kamalian S, Maas MB, et al. CT cerebral blood flow maps optimally correlate with admission diffusion-weighted imaging in acute stroke but thresholds vary by postprocessing platform. Stroke 2011;42:1923-28

8. Kamalian S, Kamalian S, Konstas AA, et al. CT perfusion mean transit time maps optimally distinguish benign oligemia from true "atrisk" ischemic penumbra, but thresholds vary by postprocessing technique. AJNR Am J Neuroradiol 2012;33:545-49

9. Jenkinson M, Beckmann CF, Behrens TEJ, et al. FSL. Neuroimage 2012;62:782-90

10. Smith SM. Fast robust automated brain extraction. Hum Brain Mapp 2002;17:143-55

11. Jenkinson $M$, Bannister $P, B$ Bady $M$, et al. Improved optimization for the robust and accurate linear registration and motion correction of brain images. Neuroimage 2002;17:825-41

12. Rorden C, Brett M. Stereotaxic display of brain lesions. Behav Neurol 2000;12:191-200

13. Schellinger PD, Bryan RN, Caplan LR, et al. Evidence-based guideline: the role of diffusion and perfusion MRI for the diagnosis of acute ischemic stroke-report of the Therapeutics and Technology Assessment Subcommittee of the American Academy of Neurology. Neurology 2010;75:177-85

14. Wintermark M, Albers GW, Alexandrov AV, et al. Acute stroke imaging research roadmap. AJNR Am J Neuroradiol 2008;29:e23-30

15. Wintermark M, Albers GW, Broderick JP, et al. Acute stroke imaging research roadmap II. Stroke 2013;44:2628-39

16. Bae KT. Intravenous contrast medium administration and scan timing at CT: considerations and approaches. Radiology 2010; 256:32-61
17. Konstas AA, Goldmakher GV, Lee T-Y, et al. Theoretic basis and technical implementations of CT perfusion in acute ischemic stroke. Part 2. Technical implementations. AJNR Am J Neuroradiol 2009;30:885-92

18. Murphy BD, Fox AJ, Lee DH, et al. Identification of penumbra and infarct in acute ischemic stroke using computed tomography perfusion-derived blood flow and blood volume measurements. Stroke 2006;37:1771-77

19. Powers WJ, Grubb RL, Raichle ME. Physiological responses to focal cerebral ischemia in humans. Ann Neurol 1984;16:546-52

20. Maulaz A, Piechowski-Józwiak B, Michel P, et al. Selecting patients for early stroke treatment with penumbra images. Cerebrovasc Dis 2005;20(suppl 2):19-24

21. Kudo K, Sasaki M, Yamada K, et al. Differences in CT perfusion maps generated by different commercial software: quantitative analysis by using identical source data of acute stroke patients. $\mathrm{Ra}$ diology 2010;254:200-09

22. Dohmen C, Kumura E, Rosner G, et al. Adenosine in relation to calcium homeostasis: comparison between gray and white matter ischemia. J Cereb Blood Flow Metab 2001;21:503-10

23. Helenius J, Perkiö J, Soinne L, et al. Cerebral hemodynamics in a healthy population measured by dynamic susceptibility contrast MR imaging. Acta Radiol 2003;44:538-46

24. Stys PK, Ransom BR, Waxman SG, et al. Role of extracellular calcium in anoxic injury of mammalian central white matter. Proc Natl Acad Sci U S A 1990;87:4212-16

25. Arakawa S, Wright PM, Koga M, et al. Ischemic thresholds for gray and white matter: a diffusion and perfusion magnetic resonance study. Stroke 2006;37:1211-16 